\title{
artigo
}

Peters, A.A.; Jeremias, J.S.; Cordeiro, G.F.T.; Brugger, E.B.A.; Costa, R.C.A.; Assis, G.P.; Oliveira, E.C.;

Assistência de enfermagem a pessoas com transtorno mental no hospital geral: desafios do cuidado especializado

\section{Assistência de enfermagem a pessoas com transtorno mental no hospital geral: desafios do cuidado especializado}

\author{
Nursing care for people with mental disorder in the general hospital: challenges of specialized care
}

Atención de enfermería para personas con trastorno mental en el hospital general: desafíos de la atención especializada

\begin{abstract}
RESUMO
Objetivo: Analisar a percepção e os desafios concebidos pela equipe de enfermagem, no atendimento especializado a pessoa com transtorno mental, no âmbito hospitalar. Metodologia: Estudo de campo do tipo descritivo-exploratório com abordagem da perspectiva qualitativa de produção e análise de dados. Resultados: Emergiram duas categorias temáticas: a) Percepção da equipe de enfermagem em relação à pessoa com transtorno mental no âmbito hospitalar b) Desafios da enfermagem para assistir a pessoa com transtorno mental. 0 estudo desvela os desafios que os profissionais de enfermagem encontram para assistir os pacientes com transtornos mentais no hospital geral, ainda que compreendam que essas pessoas são passíveis de agressão, reconhecem que através das práticas alicerçadas na abordagem humanizada, é possível promover relações de confiança e respeito à subjetividade de cada indivíduo, resultando na promoção de autonomia dos indivíduos envolvidos no processo de cuidado. Conclusão: A partir destas reflexões, observa-se a importância do estabelecimento de vínculo capaz de estimular a confiança entre os profissionais de enfermagem e pacientes, proporcionando a ampliação das práticas psicossociais.
\end{abstract}

DESCRITORES: Assistência de Enfermagem; Enfermagem Psiquiátrica; Saúde Mental; Hospital Geral.

\section{ABSTRACT}

Objective: To analyze the perception and challenges conceived by the nursing team, in specialized care for people with mental disorders, in the hospital environment. Methodology: This is a descriptive-exploratory field study with a qualitative perspective of data production and analysis. Results: Two thematic categories emerged: a) Perception of the nursing team in relation to the person with mental disorder in the hospital environment b) Nursing challenges to assist the person with mental disorder. The study unveils the challenges that nursing professionals encounter to assist patients with mental disorders in the general hospital, even though they understand that these people are susceptible to aggression, they recognize that through practices based on the humanized approach, it is possible to promote relationships of trust and respect to the subjectivity of each individual, resulting in the promotion of autonomy of the individuals involved in the care process. Conclusion: From these reflections, it is possible to observe the importance of establishing a bond capable of stimulating trust between nursing professionals and patients, providing the expansion of psychosocial practices.

DESCRIPTORS: Nursing Assistance; Psychiatric Nursing; Mental Health; General Hospital.

\section{RESUMEN}

Objetivo: Analizar la percepción y los desafíos concebidos por el equipo de enfermería, en atención especializada para personas con trastornos mentales, en el entorno hospitalario. Metodología: Estudio de campo descriptivo-exploratorio con una perspectiva cualitativa de producción y análisis de datos. Resultados: Surgieron dos categorías temáticas: a) Percepción del equipo de enfermería en relación con la persona con trastorno mental en el entorno hospitalario b) Desafíos de enfermería para ayudar a la persona con trastorno mental. El estudio revela los desafíos que enfrentan los profesionales de enfermería para ayudar a los pacientes con trastornos mentales en el hospital general, aunque entienden que estas personas son susceptibles a la agresión, reconocen que a través de prácticas basadas en el enfoque humanizado, es posible promover relaciones de confianza y respeto a la subjetividad de cada individuo, lo que resulta en la promoción de la autonomía de los individuos involucrados en el proceso de atención. Conclusión: A partir de estas reflexiones, es posible observar la importancia de establecer un vínculo capaz de estimular la confianza entre los profesionales de enfermería y los pacientes, proporcionando la expansión de las prácticas psicosociales.

DESCRIPTORES: Asistencia de Enfermería; Enfermería Psiquiátrica; Salud Mental; Hospital General.

RECEBIDO EM: 03/06/2020 APROVADO EM: 03/06/2020 
Angela Aparecida Peters

Enfermeira. Doutoranda pela Escola de Enfermagem Anna Nery da Universidade Federal do Rio de Janeiro. RJ, Brasil. ORCID:0000-0002-0363-8371.

\section{Juliana da Silva Jeremias}

Enfermeira. Graduada em Enfermagem pela Faculdade de Ciências Médicas - Suprema. MG, Brasil.

ORCID: 0000-0002-1494-2678

\section{Gisele Fernandes Tarma Cordeiro}

Enfermeira. Especialista em Unidade de Terapia Intensiva Adulto e Neonatal. Doutoranda em Enfermagem pela Escola de Enfermagem Anna Nery da Universidade Federal do Rio de Janeiro. RJ, Brasil.

ORCID: 0000-0001-5083-6373

\section{Érika Bicalho de Almeida Brugger}

Enfermeira. Doutora em Enfermagem. Docente. Faculdade de Ciências Médicas - Suprema. MG, Brasil.

ORCID: 0000-0002-9307-9437

\section{Rita de Cássia Almeida da Costa}

Enfermeira. Mestre em Educação pela Universidade Católica de Petrópolis- RJ, Docente na Faculdade de Ciências Médicas Suprema. MG, Brasil.

ORCID: 0000-0001-7974-2848

\section{Giselle de Paula Assis}

Enfermeira. Especialista em Saúde Mental e Mestranda do Programa de Pós-graduação em Saúde Coletiva da Universidade Federal de Juiz de Fora. MG, Brasil.

ORCID: 0000-0002-5105-4960

\section{Ernani Coimbra de Oliveira}

Enfermeiro. Doutorado em Saúde mental, docente no Instituto Federal de Educação, Ciência e Tecnologia do Sudeste de Minas Gerais. Universidade Federal Fluminense. RJ, Brasil.

ORCID: 0000-0002-6694-6427

\section{INTRODUÇÃO}

$\mathbf{N}$ o Brasil, concomitantemente com o movimento da Reforma Sanitária em que lutas foram instituídas para a garantia dos direitos à saúde da população, foi fortalecido o movimento de Reforma Psiquiátrica (RP) que buscava humanização do atendimento das pessoas com transtorno mental, propondo a internação de longa permanência como último recurso terapêutico, em que todas as alternativas extra-hospitalares se fizessem insuficientes. A garantia de direitos das pessoas com transtorno mental foi legalmente sancionada através da Lei n. ${ }^{\circ} 10.216$, em 6 de abril de 2001, provocando reflexões que repercutiram em mudanças nas diferentes regiões do país, após a divulgação de críticas contundentes ao modelo manicomial ${ }^{(1)}$.

Para garantir a continuidade do cuidado em sociedade das pessoas com transtornos mentais, a Portaria do Ministério da Saúde n. ${ }^{\circ}$ 3.088/11 instituiu a Rede de Atenção Psicossocial (RAPS), que norteia o funcionamento do atendimento psicossocial em rede de maneira articulada, determinando a atenção em saúde mental nos seguintes dispositivos: Atenção Básica em Saúde, Atenção Psicossocial Especializada, Atenção de Urgência e Emergência, Atenção de Residência de Caráter Transitório, Atenção Hospitalar, bem como, estratégias de desinstitucionalização e reabilitação psicossocial ${ }^{(2)}$.

No contexto do cuidado psiquiátrico e da atenção em saúde mental em hospitais gerais, o movimento de RP ampliou a ênfase na possibilidade de tratamento para as necessidades físicas e psíquicas no mesmo espaço de outros pacientes assistidos pela mesma equipe por meio da criação das unidades de psiquiatria (UP), sendo estes leitos direcionados ao atendimento de pessoas com transtornos mentais ${ }^{(3)}$.
Nessa perspectiva, o processo de integração entre a psiquiatria e outras especialidades nesses espaços vem ocorrendo de forma contínua e complexa, ainda que de forma tímida e incipiente, quando comparados ao que ocorre com outras clínicas nesse contexto do hospital, tendo que superar muitas resistências e transposição de modelos de cuidados, especialmente o proposto pela medicina, que quase sempre resulta no tratamento centrado em terapêuticas somáticas, portanto, restringindo seu escopo de possibilidades ${ }^{(4)}$.

É certo que a RP já conseguiu inspirar diversas e importantes transformações nas práticas e no cotidiano da enfermagem psiquiátrica, a qual tem contribuído para melhorar a qualidade da atenção ao paciente pelo uso de habilidades, tais como: acolhimento, empatia e escuta ativa, dentre outras perscrutadas pela e na oferta de cuidados de enfermagem. Diante desta 
nova fase de reestruturação da assistência, os atores envolvidos são convocados a pensarem em seus novos papéis em relação ao cuidado de pessoas com transtornos mentais, levando à dedicação ao desenvolvimento de propostas para práticas fundamentadas no conhecimento técnico-científico, ético e humanizado ${ }^{(5-6)}$.

Os profissionais de enfermagem que atuam na assistência direta e com contato constante e intenso com o paciente com transtornos mental, tendem, por meio da vivência dessas experiências, acumular um saber sensorial. Neste cenário, discussões atuais são de suma importância para a construção do fortalecimento do papel da equipe de enfermagem, que passou a desempenhar atividades a partir de um novo olhar, com atenção à subjetividade de cada sujeito em seu meio social, o qual envolve os familiares e a equipe multidisciplinar $^{(7-8)}$.

Este estudo se justifica por ser pertinente no campo da assistência em saúde mental. Pesquisas que desvelam debates acerca da qualificação dos profissionais de enfermagem na área, pois, de certa forma, faz uma valiosa ressonância por meio do acesso às narrativas de profissionais envolvidos com o cuidado de pessoas com transtornos mentais no hospital geral, desnudando as dificuldades com as quais eventualmente estão envolvidos nas relações de cuidado.

Neste sentido, o estudo contribuirá para a superação de dificuldades vivenciadas pelas equipes de saúde diante da percepção das fragilidades encontradas no cenário estudado, visando ações que possam contemplar a assistência ao paciente com transtorno mental no hospital geral.

O objetivo deste estudo foi analisar a percepção e os desafios concebidos pela equipe de enfermagem no atendimento especializado à pessoa com transtorno mental, no âmbito hospitalar.

\section{METODOLOGIA}

Estudo de campo do tipo descritivo-exploratório com abordagem da perspectiva qualitativa de produção e análise de dados. As metodologias de pesquisa qualitativas podem ser entendidas como aquelas capazes de incorporar a questão do significado e da intencionalidade como inerentes aos atos, às relações humanas e às estruturas sociais como construções humanas significativas ${ }^{(9)}$.

\section{O objetivo deste}

estudo foi analisar a

percepção e os desa-

fios concebidos pela

equipe de enferma-

gem no atendimento

especializado à pes-

soa com transtorno

mental, no âmbito

hospitalar.

A etapa de campo foi conduzida nos meses de abril e maio de 2018, com entrevista aberta com 11 profissionais da equipe de enfermagem que atuam no hospital em um Município de Minas Gerais, cujo a razão social de cadastro junto ao SUS é Psiquiatria e, tradicionalmente, o hospital é caracterizado por atender pacientes psiquiátricos. $\mathrm{O}$ atendimento prestado pelo hospital é dividido em enfermarias, sendo que a maior parte dos leitos são públicos (SUS) e uma pequena parte são leitos privados. A distribuição dos leitos segue o seguinte critério: enfermaria de pacientes crônicos, clínico geral, pacientes psiquiátricos e enfermaria de dependência química. O hospital conta, ao todo, com aproximadamente 268 leitos.

Os depoimentos foram gravados em mídia eletrônica pelas próprias pesquisadoras, em horário previamente agendado fora do horário de trabalho, em ambiente reservado para que a privacidade fosse mantida (consultório), estabelecendo uma relação empática entre a pesquisadora e participantes, com duração aproximada de 30 minutos, guiado por um roteiro semiestruturado com perguntas que tendenciavam a responder o objetivo da pesquisa.

Os riscos para a pesquisa foram mínimos, os depoentes tiveram a garantia de retirar sua entrevista da pesquisa a qualquer momento, sem que isso lhe causasse qualquer constrangimento, prejuízo ou danos. Como critérios de inclusão, foram técnicos de enfermagem e enfermeiros que trabalham na instituição no período superior a 3 meses e excluídos os que estavam de férias ou licenciados por algum motivo. Um pseudônimo foi indicado pela letra " $E$ " para enfermeiros e " $T$ " para técnicos de enfermagem e na sequência alfanumérica em que ocorreram as entrevistas (E1, E2 e/ ou T1, T2...), assim por diante para identificação do participante. Estes somente responderam as questôes após esclarecidos os objetivos do estudo, compreendido e assinado o Termo de Consentimento Livre e Esclarecido (TCLE). A coleta de dados não implicou em alterações da rotina de trabalho dos colaboradores na Instituição.

Os dados foram analisados por meio da técnica de categorização de Bardin ${ }^{(10)}$, composta por três grandes etapas: pré-análise; fase de organização, onde ocorreu a leitura flutuante e elaboração de indicadores que fundamentaram a interpretação; exploração do material, as unidades de registro (identificadas na etapa anterior) foram organizadas visando à categorização; e tratamento dos resultados que compreendeu a inferência e a interpretação.

Posteriormente, as entrevistas foram transcritas na íntegra e disponibilizadas para leitura e autorização dos participantes, para que estes tivessem o livre arbítrio de omitir alguma informação ou acrescentar algo que pudesse contribuir para o estudo proposto.

O projeto de pesquisa foi analisado pelo Comitê de Ética da Faculdade de Ciências 
Médicas e Saúde de Juiz de Fora - MG SUPREMA sendo deferido como aprovado segundo Parecer de número 2.570.658, conforme preconizado pela Resolução n. ${ }^{\circ}$ 466/2012, do Conselho Nacional de Saúde, que regulamenta a pesquisa com seres humanos.

\section{RESULTADOS}

Participaram desta pesquisa 11 profissionais de enfermagem, sendo seis técnicos e cinco enfermeiros. Com tempo de formação de seis meses a 23 anos para os enfermeiros, e em relação aos técnicos de enfermagem, de três a 20 anos. Em relação aos profissionais enfermeiros, todos apresentavam Curso de Especialização em áreas diversas, porém nenhum com Especialização em Enfermagem Psiquiátrica e Saúde Mental.

Através da análise do material, foi possível identificar as seguintes categorias relacionadas às vivências da equipe de enfermagem na atenção ao paciente com transtorno mental no ambiente hospitalar:

Percepção da equipe de enfermagem em relação a pessoa com transtorno mental no âmbito hospitalar

Os recortes a seguir, revelam iniciativas que potencializam as práticas de valorização da autonomia e autocuidado das pessoas com transtornos mentais:

[...] alguns são agressivos, outros são compreensivos, tem dificuldade de realizar suas próprias tarefas cotidianas, as vezes eles chegam surtados, depois normaliza, depende de cada quadro[...] $(T, 6)$

Os pacientes se tornam agressivos com palavras, força física, não chegam a agredir, mas não permitem que chegue perto, ou até mesmo conversar com ele, mais muitos pacientes são cooperativos $[\ldots](T, 4)$

[...] alguns pacientes são agressivos outros são totalmente dependentes por parte da enfermagem, não conse- guem fazer suas atividades sozinhos, mas o paciente doente mental em si, é um paciente tranquilo, não dá trabalho, carinhoso no seu dia-dia éum paciente tranquilo[...] $(E, 3)$

Hoje pelo o fato de trabalhar muito tempo com esses pacientes já consigo ter um olhar diferenciado em relação a eles, as vezes ele não está tão agressivo, ele está apenas mais confuso, assim cada momento com o paciente, tenho que ter um cuidado diferenciado em relação a ele [...] (E,5)

\section{Percepção quanto aos cuidados}

Opaciente com algum tipo de transtorno mental tem dificuldade de saber a importância de se escovar os dentes, de se alimentar, é um cuidado que a enfermagem tem que está mais próxima do paciente, olhando se ele está comendo, dormindo, se está agitado ou se está apresentando mais alguma coisa [...] $(E, 1)$

O primeiro cuidado tem que ser o acolbimento, é preciso abordar de forma humanizada, ver quais são as necessidades dele, como esse paciente se encontra naquele momento, para gente tentar trabalhar de uma forma acolhedora, humaniza$d a$, e vê-lo como único e não como mais um na instituição [...] $(E, 3)$

Nós vamos auxiliando e mostrando como realizar as tarefas principais [...] é muito importante incentivar eles a realizarem sozinhos, se eles se alimentam devagar, vamos devagar para ele ter liberdade. Inclusive, ontem teve um paciente muito agressivo, eu fiz um passeio com ele, porque o paciente ficar só dentro da enfermaria não dá, ele tem de vivenciar um pouco de lazer [...] $(T, 6)$

Desafios da enfermagem para assistir a pessoa com transtorno mental

Os trechos a seguir demonstram que a enfermagem encontra dificuldades na assistência em saúde mental, devido a características presentes em alguns transtornos, como: alteração no comportamento, agressividade e irritabilidade.

O mais difícil é o primeiro contato com o paciente, pois é preciso fazer com que este tenha confiança em quem está lidando com ele, ou seja, a equipe. Nem sempre este é um papel fácil, são pessoas fora de seu convivio, há então um processo de conquistar a confiança do mesmo [...] $(E, 4)$.

É um desafio o primeiro contato com o paciente, pois podemos oferecer um certo conforto e segurança que of familiar não consegue identificar, porque a gente acaba se aproximando do paciente com transtorno mental $[. .].(E, 2)$

Há uma preocupação e uma dificuldade maior com os pacientes com transtorno mental, pois em muitos casos ele é agressivo, e para tanto é preciso medicá-lo, deixando-o mais tranquilo. Chega muito paciente em crise, com esquizofrenia, transtorno afetivo bipolar, tem vários pacientes que chegam com algum transtorno, são pacientes que chegam aqui para a gente descompensado $[. .].(E, 5)$

Os pacientes quando chegam agitados é um desafio, mas a conversa é uma das melhores opções [...] $(T, 5)$

\section{DISCUSSÃO}

Os profissionais da equipe de enfermagem percebem a pessoa com transtornos mentais como uma pessoa passível de agressão, expectativas que nos levam a crer que os manicômios brasileiros do século XX não apenas descredenciaram socialmente sob a inscrição da loucura, pessoas que por eles passaram, mas também construíram experiências que, na atualidade, ainda influenciam negativamente 
nas formas com as quais os corpos dos sujeitos cuidados se relacionam com suas demandas nos espaços dos novos serviços psiquiátricos substitutivos ao manicômio, e produzem nos agentes que cuidam expectativas, por vezes, desvinculadas de uma nova realidade, conforme se identificou pelos excertos ilustrativos dos depoimentos dos profissionais de enfermagem participantes do estudo em tela ${ }^{(11)}$.

É como se a desinstitucionalização inspirada pela (RPB), em meados da década de oitenta, tivesse acontecido exclusivamente pela saída das pessoas dos antigos espaços de cerceamento e suplício, não produzindo as desejadas mudanças sociais que alterassem a forma de ver e se relacionar com as pessoas com transtornos mentais, desvelando, assim, a manutenção da institucionalização social da loucura, que insiste em regular e disciplinar esses corpos, mas de forma silenciada e hiperespecializada, como pode-se verificar pelos relatos dos profissionais de enfermagem participantes do estudo a respeito do funcionamento na interação com a pessoa com transtornos mentais durante as relações de cuidado nas enfermarias de leito psiquiátrico em hospital geral ${ }^{(8-12)}$.

Entretanto, esses mesmos profissionais que apresentaram, pelos excertos ilustrativos das entrevistas nas categorias anteriores, expectativas estereotipadas a respeito das possibilidades previstas na interação com a pessoa com transtornos mentais na relação de cuidado, também reconhecem que, através das práticas alicerçadas na abordagem humanizada, é possível promover relações de confiança e respeito à subjetividade de cada indivíduo, resultando na promoção de autonomia dos indivíduos envolvidos no processo de cuidado ${ }^{(12)}$.

Neste sentido, é importante que o profissional compreenda que, para além da alta na instituição psiquiátrica, o conceito de desinstitucionalização engloba o abandono de propostas da psiquiatria tradicional, e impõe a incorporação de conceitos de ações comunitárias e socializantes na prática assistencial dos profissionais, que devem assistir as pessoas com transtornos mentais e seus familiares, fora da lógica de
E para além do olhar

clínico, é possível

identificar o cuidado

de enfermagem

orientado

pelas práticas

biopsicossociais,

quando se referem

à importância

do acolhimento,

da humanização

da assistência e

possibilidade

de produção de

autonomia dos

clientes através

de uma relação

de respeito às

subjetividades

dessas pessoas que

demandam cuidados

no âmbito hospitalar. isolamento e opressão, tendo como objetivo reduzir o estigma referente à assistência. Assim, a desinstitucionalização e o resgate da cidadania, através de práticas e saberes, têm como foco principal a percepção do indivíduo em sua singularidade ${ }^{(11-12)}$.

Os profissionais de enfermagem através dos relatos expressam que reconhecem as especificidades da assistência ao paciente que apresenta transtorno mental, quando apresentam o olhar sensibilizado às demandas de cada pessoa, observando se estão se alimentando, padrão de sono, acompanhando e auxiliando nas atividades de vida diária. E para além do olhar clínico, é possível identificar o cuidado de enfermagem orientado pelas práticas biopsicossociais, quando se referem à importância do acolhimento, da humanização da assistência e possibilidade de produção de autonomia dos clientes através de uma relação de respeito às subjetividades dessas pessoas que demandam cuidados no âmbito hospitalar. Esses aspectos são fundamentais para RP, pois propicia reflexões sobre o compromisso de reconhecer o outro como sujeito único, em seu meio social, através da ressignificação do cuidar em saúde mental, fora de rotulações estigmatizantes ${ }^{(13)}$.

Assim, o acolhimento, considerado como uma tecnologia de cuidado para humanização da assistência, foi construído e inserido na relação profissional, usuário e sociedade, o qual favoreceu a construção de relações de confiança e compromisso entre a equipe de saúde multiprofissional e usuários, proporcionando a esses indivíduos a potencialização da participação ativa em seu contexto de atendimento em saúde. A partir de relações alicerçadas no respeito mútuo, em que a construção da autonomia de cada indivíduo fica mais sutil, através da responsabilização compartilhada e pactuada entre os sujeitos envolvidos no cuidado terapêutico ${ }^{(14)}$.

Fica claro nas assertivas dos profissionais que o vínculo promovido pela equipe de enfermagem ao paciente com transtorno mental favorece a integralidade da atenção à saúde. Revela-se a importância de ouvir o próximo em suas necessidades e de se fazer ouvido, através do diálogo. $\mathrm{O}$ 
vínculo se constitui a partir de um instrumento de trabalho que estabelece trocas de saberes entre o técnico e o popular, o científico e o empírico, o objetivo e o subjetivo, o qual promove a construção de ações terapêuticas estabelecidas a partir das características de cada coletivo e de cada indivíduo ${ }^{(15)}$.

Cabe destacar que, essas ações são de extrema importância à saúde integral dos sujeitos que se encontravam desprovidos de sua dignidade, bem como de seus direitos humanos, pela subjugação ao modelo de tratamento asilar em manicômios, que geravam a segregação da doença e invisibilidade social.

Quando se estabelece a reformulação do cuidado em saúde mental a partir da $\mathrm{RP}$, os profissionais de enfermagem passam a atuar no exercício autônomo da profissão, e se responsabilizam pela assistência através de relações democráticas. Nessa perspectiva, além do acompanhamento referente ao diagnóstico, identificação de sinais e sintomas, cuidados com higiene e administração de medicamentos, esses profissionais ampliam suas atividades através de propostas terapêuticas pelo modelo de atenção psicossocial, primando pelo cuidado inclusivo da vida humana, para que ocorra a ampliação do foco do cuidado em seus aspectos biopsicossociais ${ }^{(16)}$.

A partir dessas circunstancias, é pertinente enfatizar que os profissionais que assistem em saúde mental estão diariamente desenvolvendo estratégias de reabilitação psicossocial e resgate da autonomia, pois, na medida em que trabalham com o olhar voltado para o sujeito em toda sua complexidade e se familiarizam com o sofrimento e as fragilidades do ser humano, colocam em prática estratégias para a reabilitação psicossocial.

A relação de confiança com o paciente com transtorno mental é um processo complexo no qual a abordagem centrada na pessoa, é um modo de cuidar que transcende o paradigma tradicional da psiquiatria e se concretiza por meio da relação terapêutica e ajuda o paciente a expressar melhor seus sentimentos. Destarte, é possível perceber nas entrelinhas dos depoimentos a estigmatização da doença mental, fazendo com que a equipe de enfermagem deste estudo sofra com as atribuiçôes reducionistas do seu saber ${ }^{(7-17)}$.

Ao expressar que a enfermagem no estabelecimento do cuidado acaba se aproximando desses clientes, é importante a percepção de que a proximidade deveria ser algo natural, fora de visóes estereotipadas, para que a disponibilidade da equipe de enfermagem, em se atentar às particularidades que envolvem a assistência especializada e o saber ouvir, estabeleça relações de respeito com respostas de adequação à atenção psicossocial no ambiente hospitalar, que não deve ser visto por essas pessoas como espaço de repressão, e sim de liberdade e inclusão de suas demandas ${ }^{(7-18)}$.

Cabe destacar que, a ética é um fator fundamental para o profissional que atende ou recepciona essas pessoas, visto que, na maior parte dos casos, encontram-se inquietos e inseguros, muitos até mesmo agressivos, além de estarem, em alguns casos, incapazes de responder por seus atos, ficando, muitas vezes vulneráveis e/ou desorientados. Pelas colocações dos participantes, foi possível identificar a dificuldade em lidar com os pacientes que se apresentam em crise e, consequentemente, agitados por decorrência de seu quadro em alguns momentos ${ }^{(18)}$.

Sob essa concepção, é vislumbrado a terapêutica medicamentosa como um cuidado que traz tranquilidade para o assistido. É necessário discutir que a medicalização dos sintomas em quadros psiquiátricos vem sendo pouco questionada a nível global, resultando no aumento desordenado da contenção química como recurso de cuidado, e se apresenta como uma prática que gera retrocesso do paradigma de atenção psicossocial quando não necessário, e é tomado como usual ${ }^{(19-20)}$.

Ainda nessa perspectiva, contraponto à medicalização da doença mental, foi relatado que a conversa ainda é compreendida como melhor opção terapêutica para o cuidado de enfermagem. Inferimos que o diálogo é sempre um facilitador entre a pessoa que recebe os cuidados e a equipe de enfermagem, sendo a forma mais efi- caz de comunicação entre os indivíduos. Quando este se dá através do respeito mútuo, apresenta progresso e sucesso no contato entre ambos ${ }^{(20-21)}$.

Contudo, é necessária a compressão de que quando os profissionais de enfermagem, como componentes fundamentais da equipe multidisciplinar, assistem a demanda de cuidados em saúde mental sem conhecimento técnico científico, tendem a reproduzir práticas reducionistas que estão na contramão do paradigma de cuidado psicossocial. Portanto, é necessária a capacitação e busca contínua pela qualificação profissional em consonância do que é contemplado pela RP, para que ocorra de fato a ampliação do cuidado humanizado, para além do discurso do que deveria ser o ideal ${ }^{(20)}$.

\section{CONCLUSÃO}

As mudanças propostas pela RPB trouxeram para os profissionais de enfermagem uma nova fase, em que não se limita à prática da administração de medicamentos, higiene e alimentação, pelo contrário, atuam como mediador entre o indivíduo e sua família. Os colaboradores da presente pesquisa reconhecem que o cuidado em saúde mental envolve fatores condicionantes à qualidade de vida, dentre eles pode-se citar: alimentação, educação, moradia, lazer e o convívio familiar. Relatam que essas pessoas necessitam de atenção e cuidados diferenciados pela equipe que os assistem.

Observamos na realização deste estudo, a necessidade de investimento em relação à capacitação para melhoria dos cuidados de enfermagem à pessoa com transtorno mental no hospital geral, de modo a lhes assegurar melhor qualidade de vida. Dentre estes, ressalta-se a necessidade de os enfermeiros se atualizarem a fim de ampliar suas competências, adaptando-se às novas modalidades de tratamento.

Do exposto, entendemos que a Reforma Psiquiátrica trouxe melhorias na atenção ao paciente com transtorno mental, passando de um atendimento individual com foco na doença, para 


\section{artigo}

Peters, A.A.; Jeremias, J.S.; Cordeiro, G.F.T.; Brugger, E.B.A.; Costa, R.C.A.; Assis, G.P.; Oliveira, E.C.

Assistência de enfermagem a pessoas com transtorno mental no hospital geral: desafios do cuidado especializado

um atendimento que considera a singularidade do sujeito e o não isolamento, tendo no espaço de internação no hospital geral, que ocupa lugar como dispositivo da RAPS, possibilidades de desenvolver relacionamentos afetivos, familiares e sociais.
Mesmo observando que na prática há falta de especialização em saúde mental, verificamos que o trabalho cotidiano proporciona um conhecimento entre os profissionais. Percebe-se então, em cada momento, a possibilidade de reconstruir novas maneiras de cuidar, possibilitando ao paciente que, demanda cuidados em saúde mental, seja reconhecido em suas particularidades, respeitando o direito ao exercício de cidadania. Essa visão faz toda a diferença para o paciente e amplia o paradigma de atenção psicossocial nos dispositivos de atenção substitutivos ao manicômio.

\section{REFERÊNCIAS}

1. Moll MF, et al. Profissionais De Enfermagem e a Internação Psiquiátrica Em Hospital Geral: Percepções E Capacitação Profissional. Cogitare Enfermagem. 2017 ju;22(2). doi: 10.5380/ce. v22i2.49933

2. Ministério da Saúde, Secretaria de Atenção à Saúde, Departamento de Atenção Básica (BR). Saúde mental / Ministério da Saúde, Secretaria de Atenção à Saúde, Departamento de Atenção Básica, Departamento de Ações Programáticas Estratégicas. Brasília (DF): MS, 2013. 176 p.: il. (Cadernos de Atenção Básica, ก. 34)

3. Ministério da Saúde (BR). Portaria n. ${ }^{\circ}$ 2.840/GM, de 29 de dezembro de 2014. Cria o Programa de Desinstitucionalização integrante do componente Estratégias de Desinstitucionalização da Rede de Atenção Psicossocial (RAPS), no âmbito do Sistema Único de Saúde (SUS), e institui o respectivo incentivo financeiro de custeio mensal. Diário Oficial da União. Brasília (DF): MS, 2014.

4. Botega NJ. Prática psiquiátrica no hospital geral: Interconsulta e emergência. Porto Alegre: Artmed; 2017.

5. Pitta AMF, Guljor AP. The violance of the counter-psychiatric reform in Brazil: on attack on democracy in times of struggle for Humanos Rights and social justice. Cadernos do CEAS: Revista Crítica de Humanidades. 2019;246:6-14. doi: 10.25247/2447861X.2019.n246.p6-14

6. Pitta AMF, Guljor AP. The violance of the counter-psychiatric reform in Brazil: on attack on democracy in times of struggle for Humanos Rights and social justice. Cadernos do CEAS: Revista Crítica de Humanidades. 2019;246:6-14. doi: 10.25247/2447861X.2019.n246.p6-14

7. Silva MS, Machado PAT, Nascimento RS, Oliveira TS, Silva TF, Batista EC. Nursing in the field of mental health: a brief theoretical discussion. Rev Amazônia Science \& Health. 2017;5(2):40-46. doi: http://10.0.72.174/2318-1419

8. Rodrigues AAP, Figueiredo MAG, Filho AJA, Peres MAA. Estratégias de ampliação do saber/poder da equipe de enfermagem em uma clínica psiquiátrica. Rev. Hist. enferm. 2014;5(2):205-205.

9. Minayo MCS. O desafio do conhecimento: pesquisa qualitativa em saúde. Ed. 14. São Paulo: Hucitec/Abrasco; 2014. p. 407.

10. Bardin L. Análise de conteúdo. São Paulo: Edições 70 Brasil; 2011.

11. Oliveira EC. Contato e desenvolvimento da presença na interação com usuários de serviços psiquiátricos: contextualização e construção discursiva. Tese de (doutorado): Jose Carlos Goncalves, orientador Universidade Federal Fluminense, Niterói, 2019.115 f. doi: 10.22409/POSLING.2019.d.05309265635

12. Amarante P. Saúde Mental e Atenção Psicossocial. Ed. 4. Rio de Janeiro: Editora Fiocruz; 2017

13. Campos DB, Bezerra IC, Jorge MSB. Produção do cuidado em saúde mental: práticas territoriais na rede psicossocial. Trab. educ. saúde. 2019;18(1):e0023167. doi: 10.1590/1981-7746sol00231

14. Carrara GLR, Moreira GMD, Facundes GM, Pereira RS, Baldo PL. Assistência de enfermagem humanizada em saúde mental: uma revisão da literatura. Rev. Fafibe [Internet]. 2015 [acesso em 20 jun 2020];8(1):86-10. Disponível em: http://unifafibe. com.br/revistasonline/arquivos/revistafafibeonline/sumario/36/30102015183642.pdf

15. Souza MC, Afonso MLM. Saberes e práticas de enfermeiros na saúde mental: desafios diante da reforma psiquiátrica. Rev. Inter. de Psicologia [Internet]. 2015 [acesso em 20 jun 2020];8(2):33247. Disponivel em: http://pepsic.bvsalud.org/pdf/gerais/v8n2/ v8n2a04.pdf

16.Santos EO, Eslabão AD, Kantorski LP, Pinho LB. Nursing practices in a Psychological Care Center. Rev Bras Enferm. 2020;73(1):e20180175. doi: http://dx.doi.org/10.1590/00347167-2018-0175

17. Alves SR, Santos RP, Oliveira RG, et al. Serviços de saúde mental: percepção da enfermagem em relação à sobrecarga e condições de trabalho. Rev Fund Care [Internet]. 2018 [acesso em 20 jun 2020];10(1):25-29. Disponivel em: https://pesquisa.bvsalud. org/portal/resource/pt/biblio-908412

18. Mota AS, Silva ALA, Souza AC. Educação permanente: Práticas e processos da enfermagem em saúde mental. Revista Portuguesa de Enfermagem de Saúde Mental. 2016;(spe4):9-16. doi: 10.19131/rpesm.0135

20.Gonçalves LLM, Campos RTO. Narrativas de usuários de saúde mental em uma experiência de gestão autônoma de medicação. Cad Saúde Pública. 2017;33(11): e00166216. doi: 10.1590/0102$311 \times 00166216$

21. Braga RB, Pegoraro RF. Internação psiquiátrica: o que as famílias pensam sobre isso?. Rev. Psicol. Saúde. 2020 abr;12(1):61-73. doi: 10.20435/pssa.v12i1.820.

22.Camillo SO, Maiorino FT. A Importância da Escuta no Cuidado de Enfermagem. Cogitare Enfermagem. 2012;17(3). doi: 10.5380/ ce.v17i3.27826. 